\title{
CYCLICAL MONOTONICITY AND THE ERGODIC THEOREM
}

\author{
MATHIAS BEIGLBÖCK
}

\begin{abstract}
It is well known that optimal transport plans are cyclically monotone. The reverse implication that cyclically monotone transport plans are optimal needs some assumptions and the proof is non-trivial even if the costs are given by the squared euclidean distance on $\mathbb{R}^{n}$. We establish this result as a corollary to the ergodic theorem.
\end{abstract}

\section{INTRODUCTION}

We consider the Monge-Kantorovich transport problem for probabilities $\mu, \nu$ on Polish spaces $X, Y$ (cf. [Vil03, Vil09]). The set $\Pi(\mu, \nu)$ of transport plans consists of all measures on $X \times Y$ with $X$-marginal $\mu$ and $Y$-marginal $\nu$. Associated to a cost function $c: X \times Y \rightarrow[0, \infty]$ and $\pi \in \Pi(\mu, \nu)$ are the transport costs

$$
\langle c, \pi\rangle=\int_{X \times Y} c(x, y) d \pi(x, y) .
$$

The Monge-Kantorovich problem is then to determine the value

$$
P_{c}:=\inf \{\langle c, \pi\rangle: \pi \in \Pi(\mu, \nu)\}
$$

and to identify an optimal transport plan $\hat{\pi} \in \Pi(\mu, \nu)$, i.e. a minimizer of (1).

A basic and important goal is to characterize minimizers through a tractable property of their support sets: a Borel set $\Gamma \subseteq X \times Y$ is cyclically monotone iff

$c\left(x_{1}, y_{2}\right)-c\left(x_{1}, y_{1}\right)+\ldots+c\left(x_{n-1}, y_{n}\right)-c\left(x_{n-1}, y_{n-1}\right)+c\left(x_{n}, y_{1}\right)-c\left(x_{n}, y_{n}\right) \geq 0$ whenever $\left(x_{1}, y_{1}\right),\left(x_{2}, y_{2}\right), \ldots,\left(x_{n}, y_{n}\right) \in \Gamma$.

A transport plan $\pi$ is cyclically monotone if it assigns full measure to some cyclically monotone set $\Gamma$.

Concerning the origins of cyclical monotonicity in convex analysis and the study of the relation to optimality we mention [Roc66, KS92, Rüs96, GM96]. Intuitively speaking, cyclically monotone transport plans resist improvement by means of cyclical rerouting and optimal transport plans are expected to have this property. Indeed, Ambrosio and Pratelli [AP03] show for general l.s.c. $c: X \times Y \rightarrow[0, \infty]$ that every optimal transport plan is cyclically monotone.

The reverse implication is more intricate. Ambrosio and Pratelli find that there exist cyclically monotone transport plans which are not optimal; notably the construction given in [AP03, Example 3.5] makes heavy use of the fact that $c$ may attain the value $\infty$. Villani [Vil03, Problem 2.25] asked whether cyclically monotone transport plans are always optimal in the case where $c$ is the squared euclidean distance on $\mathbb{R}^{n}$. This problem was resolved by Pratelli [Pra08] and SchachermayerTeichmann [ST09]: every cyclically monotone transport plan is optimal provided that $c$ is continuous or merely l.s.c. and finitely valued. ${ }^{1}$ We give a new proof to

Theorem 1. Let $c: X \times Y \rightarrow[0, \infty)$ be a measurable cost function and $\pi \in \Pi(\mu, \nu)$ a finite cost transport plan which is cyclically monotone. Then $\pi$ is optimal.

\footnotetext{
Key words and phrases. ergodic theorem, optimal transport, cyclical monotonicity.

${ }^{1}$ We refer to [BGMS09] and [BC10] for more general results, in particular lower semi-continuity
} of the cost function is not important in either implication. 


\section{Theorem 1 As Consequence of the Ergodic Theorem}

The novelty of our approach is to connect cyclical monotonicity to the pointwise ergodic theorem which we restate here: let $(Z, \kappa)$ be a probability space and $\sigma$ : $Z \rightarrow Z$ measure-preserving, i.e. $\sigma(\kappa)=\kappa$. Then for every $f \in L^{1}(\kappa)$ the limit

$$
f^{*}=\lim _{n} \frac{1}{n} \sum_{k=0}^{n-1} f \circ \sigma^{k}
$$

exists almost surely and in $L^{1}(\kappa)$ (see, e.g., [Kal02, Theorem 9.6]).

Proof of Theorem 1. Let $\pi, \tilde{\pi} \in \Pi(\mu, \nu)$ be finite cost transport plans and $\pi(\Gamma)=1$ for some cyclically monotone set $\Gamma$. We have to show that $\pi$ leads to lower costs than $\tilde{\pi}$. To this end, we specify $Z=(X \times Y)^{\mathbb{N}}$ and consider the shift mapping

$$
\sigma: Z \rightarrow Z, \quad\left(x_{i}, y_{i}\right)_{i=1}^{\infty} \mapsto\left(x_{i+1}, y_{i+1}\right)_{i=1}^{\infty} .
$$

Moreover we define the projections $P, Q: Z \rightarrow X \times Y$ by

$$
P\left(\left(x_{i}, y_{i}\right)_{i=1}^{\infty}\right):=\left(x_{1}, y_{1}\right) \text { and } Q\left(\left(x_{i}, y_{i}\right)_{i=1}^{\infty}\right):=\left(x_{1}, y_{2}\right) .
$$

The transport plans $\pi$ and $\tilde{\pi}$ give rise to a natural $\sigma$-invariant measure on $Z$ :

Lemma 2.1. There is a measure $\kappa$ on $Z$ such that $\sigma(\kappa)=\kappa, P(\kappa)=\pi, Q(\kappa)=\tilde{\pi}$.

Proof. Let $\left(\pi^{y}\right)_{y \in Y}$ be the disintegration of $\pi$ with respect to $(Y, \nu)$ and let $\left(\tilde{\pi}_{x}\right)_{x \in X}$ be the disintegration of $\tilde{\pi}$ with respect to $(X, \mu)$. Then $R(y, A):=\pi^{y}(A \times\{y\})$ and $S(x, B):=\tilde{\pi}_{x}(\{x\} \times B)$ constitute transition kernels from $Y$ to $X$ resp. from $X$ to $Y$. We identify $Z$ with the product

$$
Y^{(1)} \times X^{(1)} \times Y^{(2)} \times X^{(2)} \times \ldots
$$

where $X^{(n)}, Y^{(n)}, n \geq 1$ are copies of $X$ and $Y$. Then we consider the discrete time Markov-process with initial distribution $\nu$, and the probabilities of moving from $y \in Y^{(n)}$ to $A \subseteq X^{(n)}$ resp. from $x \in X^{(n)}$ to $B \subseteq Y^{(n+1)}$ given by $R$ resp. $S$.

The resulting distribution $\kappa$ on the space of paths $Z$ has the desired properties. (We refer to [Kal02, Chapter 7] for more details on the definition of $\kappa$.)

The map $f:=c \circ Q-c \circ P \in L^{1}(\kappa)$ satisfies $\int_{X \times Y} c d \tilde{\pi}-\int_{X \times Y} c d \pi=\int_{Z} f d \kappa$. The crucial step of our proof is that, applying the ergodic theorem to the function $f$ and integrating over (2), we have

$$
\int_{Z} f d \kappa=\int_{Z} f^{*} d \kappa=\int_{Z} \lim _{n} \frac{1}{n} \sum_{k=0}^{n-1} f \circ \sigma^{k} d \kappa .
$$

Rewriting this expression in terms of $c$ we obtain

(3) $\int_{X \times Y} c d \tilde{\pi}-\int_{X \times Y} c d \pi=\int_{Z}\left[\lim _{n} \frac{1}{n} \sum_{k=1}^{n} c\left(x_{k}, y_{k+1}\right)-c\left(x_{k}, y_{k}\right)\right] d \kappa\left(x_{i}, y_{i}\right)_{i}$.

To conclude the proof it is sufficient to show that the integrand on the right side of (3) is $\kappa$-almost surely non-negative. Note that $\kappa\left(\Gamma \times(X \times Y)^{\mathbb{N}}\right)=\pi(\Gamma)=1$. Since $\kappa$ is $\sigma$-invariant and $\Gamma^{\mathbb{N}}=\bigcap_{n \geq 1}\left(\sigma^{-n}\left(\Gamma \times(X \times Y)^{\mathbb{N}}\right)\right)$ we have $\kappa\left(\Gamma^{\mathbb{N}}\right)=1$. Thus it suffices to argue on sequences $\left(x_{n}, y_{n}\right)_{n=1}^{\infty}$ with $\left(x_{n}, y_{n}\right) \in \Gamma, n \geq 1$.

We note that the proof of Theorem 1 is immediate under the additional assumption that $c$ is bounded: in this case cyclical monotonicity of $\Gamma$ trivially implies

$$
\liminf _{n} \frac{1}{n} \sum_{k=1}^{n} c\left(x_{k}, y_{k+1}\right)-c\left(x_{k}, y_{k}\right) \geq 0 \text { whenever }\left(x_{n}, y_{n}\right)_{n=1}^{\infty} \in \Gamma^{\mathbb{N}}
$$

thus $\pi$ is optimal. 
To prove Theorem 1 in the general case fix $(\bar{x}, \bar{y}) \in \Gamma$. Given $\left(x_{i}, y_{i}\right)_{i=1}^{\infty} \in \Gamma^{\mathbb{N}}$, cyclical monotonicity of $\Gamma$ implies that

$$
c\left(\bar{x}, y_{1}\right)-c(\bar{x}, \bar{y})+\left(\sum_{k=1}^{n} c\left(x_{k}, y_{k+1}\right)-c\left(x_{k}, y_{k}\right)\right)+c\left(x_{n+1}, \bar{y}\right)-c\left(x_{n+1}, y_{n+1}\right)
$$

is non-negative for each $n$. As $c$ takes values in $[0, \infty)$ this further yields

$$
\liminf _{n}\left[\frac{1}{n}\left(\sum_{k=1}^{n}\left(c\left(x_{k}, y_{k+1}\right)-c\left(x_{k}, y_{k}\right)\right)+\frac{c\left(x_{n+1}, \bar{y}\right)}{n}\right] \geq 0 .\right.
$$

Setting $g\left(\left(x_{i}, y_{i}\right)_{i=1}^{\infty}\right):=c\left(x_{1}, \bar{y}\right)$ we have $c\left(x_{n+1}, \bar{y}\right)=g \circ \sigma^{n}\left(\left(x_{i}, y_{i}\right)_{i=1}^{\infty}\right)$. As $g$ is a finite function, $g / n$ tends to 0 in measure (with respect to $\kappa$ ) and because $\sigma$ is measure preserving, also $\lim _{n \rightarrow \infty} g \circ \sigma^{n} / n=0$ in measure. Passing to a subsequence if necessary, we may assume that convergence holds $\kappa$-a.s. Together with (4) this proves that (3) is non-negative and thus concludes the proof of Theorem 1.

\section{Comments}

We have used the pointwise ergodic theorem which may be somewhat tricky for readers not accustomed to ergodic theory. We note that the simpler mean ergodic theorem is also sufficient. It asserts that $\lim _{n} \frac{1}{n} \sum_{k=0}^{n-1} f \circ \sigma^{k}$ exists in $L^{p}$ whenever $f \in L^{p}, p \geq 1$, see e.g. [Kal02, Exercise 9.14]. In particular this implies that the limit in (2) exists almost surely along a subsequence $\left(n_{m}\right)_{m=1}^{\infty}$. The above proof of Theorem 1 then still applies if we argue on $n_{m}$ rather than $n$.

Using similar arguments as in [BGMS09, BC10] our approach can be used to prove even more general versions of Theorem 1 . As this does not require new ideas nor strengthens the results of [BGMS09, BC10] we do not elaborate.

\section{REFERENCES}

[AP03] L. Ambrosio and A. Pratelli. Existence and stability results in the $L^{1}$ theory of optimal transportation. In Optimal transportation and applications (Martina Franca, 2001), volume 1813 of Lecture Notes in Math., pages 123-160. Springer, Berlin, 2003.

[BC10] S. Bianchini and L. Caravenna. On optimality of $c$-cyclically monotone transference plans. C. R. Math. Acad. Sci. Paris, 348(11-12):613-618, 2010.

[BGMS09] M. Beiglböck, M. Goldstern, G. Maresch, and W. Schachermayer. Optimal and better transport plans. J. Funct. Anal., 256(6):1907-1927, 2009.

[GM96] W. Gangbo and R. McCann. The geometry of optimal transportation. Acta Math., 177(2):113-161, 1996.

[Kal02] O. Kallenberg. Foundations of modern probability. Probability and its Applications (New York). Springer-Verlag, New York, second edition, 2002.

[KS92] M. Knott and C. Smith. On Hoeffding-Fréchet bounds and cyclic monotone relations. J. Multivariate Anal., 40(2):328-334, 1992.

[Pra08] A. Pratelli. On the sufficiency of $c$-cyclical monotonicity for optimality of transport plans. Math. Z., 258(3):677-690, 2008.

[Roc66] R. Rockafellar. Characterization of the subdifferentials of convex functions. Pacific J. Math., 17:497-510, 1966.

[Rüs96] L. Rüschendorf. On c-optimal random variables. Statist. Probab. Lett., 27(3):267-270, 1996.

[ST09] W. Schachermayer and J. Teichmann. Characterization of optimal transport plans for the Monge-Kantorovich problem. Proc. Amer. Math. Soc., 137(2):519-529, 2009.

[Vil03] C. Villani. Topics in optimal transportation, volume 58 of Graduate Studies in Mathematics. American Mathematical Society, Providence, RI, 2003.

[Vil09] C. Villani. Optimal Transport. Old and New, volume 338 of Grundlehren der mathematischen Wissenschaften. Springer, 2009.

Mathias Beiglböck

FAKUltät FÜr Mathematik, Universität WieN

Nordbergstrasse 15, 1090 Wien, Austria

E-mail address: mathias.beiglboeck@univie.ac.at 